\title{
BioS, a Biotin-Induced, Stationary-Phase, and Possible LysR-Type Regulator in Sinorhizobium meliloti
}

\author{
Elke B. Heinz, ${ }^{1}$ Donald A. Phillips, ${ }^{2}$ and Wolfgang R. Streit ${ }^{1}$ \\ ${ }^{1}$ Institut für Mikrobiologie und Genetik der Universität Göttingen, Grisebachstr. 8, 37077 Göttingen, \\ Germany; ${ }^{2}$ Department of Agronomy and Range Science, University of California, Davis 95616, U.S.A. \\ Accepted 11 May 1999.
}

\begin{abstract}
Sinorhizobium meliloti 1021 produces biotin required for growth, but it also responds to external biotin signals from alfalfa plants through the bioS regulatory locus. Mutation of bioS increases biotin uptake, extends stationary phase in the presence of biotin, and impairs competitive growth in the presence of biotin. New data supporting the relevance of this gene to plant-microbe interactions show that a bioSgus $A$ reporter fusion is expressed by bacteria on plant roots, by bacteria in alfalfa root nodules, and more generally by any stationary-phase bacterial cells in the presence of biotin. Significant transcription of bioS-gusA was detected at $50 \mathrm{pM}$ biotin, and a 16-fold induction was measured in stationary-phase cells with $1 \mathrm{nM}$ biotin. A positive autoregulation of bioS is suggested by the fact that, in the presence of $10 \mathrm{nM}$ biotin, a $\operatorname{bioS}$-gus $A$ fusion in wild-type cells was transcribed at more than twice the rate of the same reporter fusion in a bioS mutant background. Analyses of primer extension products and expression studies with three different bioS-gusA promoter fusions defined major characteristics of the bioS promoter. Expression of bioS in Escherichia coli resulted in the production of a 35-kDa BioS protein with characteristics of an LysR-type regulator. Those traits included a deduced amino acid sequence containing possible helix-turn-helix DNA-binding structures and three $\mathrm{T}-\mathrm{N}_{(11)}$-A motifs upstream from the bioS gene. A regulatory role for BioS protein was supported by tests showing that a 321-bp DNA fragment containing the bioS promoter altered the electrophoretic mobility of recombinant BioS and of a corresponding band in protein extracts from $S$. meliloti 1021. The absence in BioS of any significant homology to known amino acid sequences was consistent with the fact that DNA hybridization tests with seven genera of bacteria detected bioS only in Sinorhizobium.
\end{abstract}

Additional keywords: Medicago sativa, rhizosphere.

Basic facts about biotin are well established: It is a classic vitamin cofactor that binds $\mathrm{CO}_{2}$ as bicarbonate in various carboxylase enzymes, including acetyl CoA carboxylase ( $\mathrm{Li}$ and Cronan 1993) and pyruvate carboxylase (Dunn et al. 1996; Dunn 1998); it is produced by microorganisms and plants, but not by animals; and it regulates its own synthesis at very low con-

Corresponding author: W. R. Streit, Institut für Mikrobiologie und Genetik, Universität Göttingen, Grisebachstr. 8, 37077, Göttingen, Germany; Telephone: (49) 551-393844; Fax: (49) 551-393793; E-mail: <wstreit@gwdg.de> centrations, as biotinoyl-AMP, through the birA gene (Cronan 1989). The birA gene product is a key regulatory protein that functions both as a repressor of the bio operon (Barker and Campbell 1981) and as a biotin-protein ligase that binds biotin into acetyl-CoA carboxylase (Barker and Campbell 1981). Despite the well-known biological activity of low biotin concentrations, no other biotin-regulated bacterial genes are well characterized, and no biotin-uptake system has been genetically defined.

Sinorhizobium meliloti is an agriculturally important bacterium that forms $\mathrm{N}_{2}$-fixing root nodules on alfalfa (Medicago sativa). When alfalfa plants are no longer present, the number of $S$. meliloti cells in the soil declines markedly but, as new alfalfa seeds germinate, the bacteria multiply and form new nodules (Bottomley 1992). Recent data show that plant-derived biotin is an important factor that stimulates growth of $S$. meliloti in the rhizosphere (Streit et al. 1996). A larger, rapidly growing population of $S$. meliloti contributes immediately to root nodule formation and, ultimately through $\mathrm{N}_{2}$ fixation, to superior plant growth. Early studies established that biotin is not essential but promotes growth of (Sino)Rhizobium spp. in culture (West and Wilson 1939).

The molecular mechanism by which external biotin from the alfalfa plant stimulates growth of $S$. meliloti cells is unclear. One possible mechanism involves the biotin-dependent carboxylases present in S. meliloti (Dunn 1998). However, another possible mechanism involves bioS, a biotin-inducible gene in $S$. meliloti that affects competitive growth and biotin uptake (Streit and Phillips 1997). Three phenotypic traits are associated with bioS: (i) cells mutated in bioS compete equally with wild-type cells in biotin-free medium but poorly when $40 \mathrm{nM}$ biotin is present; (ii) bioS mutants show an increased lag phase; and (iii) stationary-phase bioS mutant cells accumulate 30 to $50 \%$ more ${ }^{14} \mathrm{C}$-biotin than wild-type cells. A possible role for bioS in stationary-phase $S$. meliloti cells is further suggested by the position of the bioS locus among three genes showing homology to survival operons of other bacteria. The present study, which was initiated to characterize further the role of bioS in $S$. meliloti, establishes that the BioS protein has traits similar to LysR-type regulators and probably is involved in sensing plant-exuded biotin.

\section{RESULTS}

Expression of bioS reporter fusions.

The bioS-gusA reporter fusion constructed in Rm1021WS7G for this study was significantly more sensitive than the bioS-nptII in Rm1021-B5, which was first used to prove that 
biotin induces bioS (Streit and Phillips 1997). For example, whereas $400 \mathrm{pM}$ was the lowest concentration of biotin that markedly induced the bioS-nptII reporter, significant $(P \leq 0.05) \beta$-glucuronidase (GUS) activity was induced in Rm1021-WS7G by $50 \mathrm{pM}$ biotin (Fig. 1). Expression of bioS increased strongly with the amounts of biotin present in the medium. In the pres-

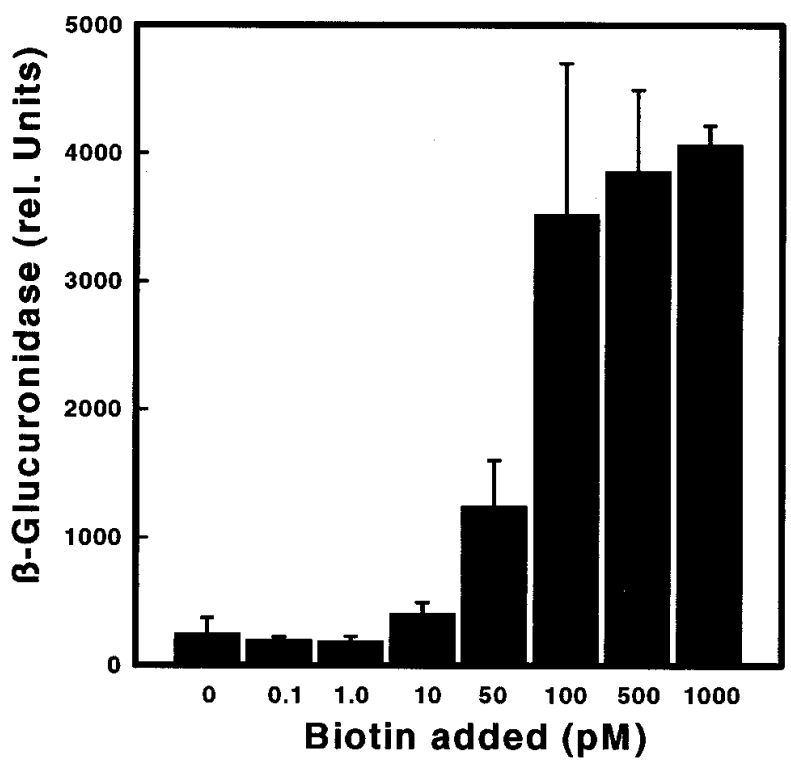

Fig. 1. Effect of low biotin concentrations on transcription of bioS in Sinorhizobium meliloti. Expression of a bioS-gusA reporter fusion was measured in stationary-phase Rm1021-WS7G cells after growth in GTS medium (Kiss et al. 1979) containing different biotin concentrations. Data represent mean \pm SD of four replicate cultures.

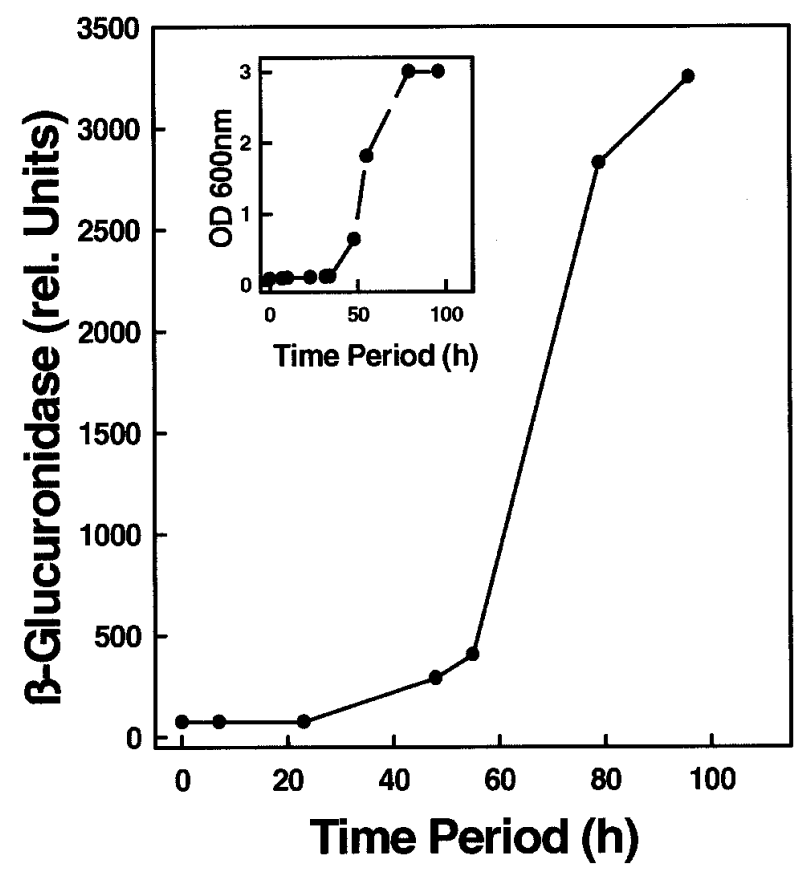

Fig. 2. Transcription of bioS in Sinorhizobium meliloti cells during growth in $10 \mathrm{nM}$ biotin. Expression of a bioS-gusA fusion measured bioS transcription in Rm1021-WS7G cells growing in GTS medium (Kiss et al. 1979). ence of $1 \mathrm{nM}$ added biotin, a 16.2-fold induction of the bioSgusA reporter fusion was measured, compared with cells that had been growing in the absence of added biotin.

Transcription of bioS in Rm1021-WS7G was greatest in stationary phase cells (Fig. 2). In total, there was a 9.2-fold increase in bioS expression between early log phase cells and those entering stationary phase. These results were confirmed with Rm1021-WSGB19, which carries Tn5-B22, a transposable promoter probe containing lacZ as a reporter. In the presence of $40 \mathrm{nM}$ biotin this mutant showed an 8.3-fold induction of the bioS-lacZ fusion when stationary phase cells were compared with cells in early log phase (Table 1). A weak expression of the bioS gene also was observed in cultures without added biotin. However, $\beta$-galactosidase activities measured in those cultures were significantly lower than in the corresponding cultures containing $40 \mathrm{nM}$ of added biotin. Correctness of the gusA and lacZ insertions in both Rm1021-WS7G and Rm1021WSGB19 was verified by polymerase chain reaction (PCR) tests and Southern hybridizations to predicted restriction fragments. In addition, the location of the promoter probe in Rm1021WSGB19 was confirmed by DNA sequencing (Fig. 3).

To examine a possible autoregulation by the BioS protein, transcriptional activity of bioS-gusA was measured in bioS mutant Rm1021-WS44G, which was constructed by homologous recombination with the bioS-gusA fusion into bioS. This mutant showed a relationship between bioS induction and growth qualitatively similar to that measured in Rm1021WS7G and Rm1021-WSGB19. However, the quantitative levels of bioS expression were generally two- to threefold lower than those detected in Rm1021-WS7G. For one such experiment in which cells grew $96 \mathrm{~h}$ in the presence of $10 \mathrm{nM}$ biotin, Rm1021-WS7G produced 3,250 relative GUS units while Rm1021-WS44G yielded 1,250 relative GUS units. The observation that bioS expression under the control of its own promoter was elevated in the bioS background is consistent with the concept that BioS regulates its own transcription.

Tests with alfalfa seed rinses, i.e., effusates that might be produced naturally during germination, supported the conclusion that biotin is the primary, perhaps only, plant-derived inducer of bioS. In those experiments, Rm1021-WSGB19 showed no induction of the bioS-lacZ in plates containing X-Gal (5bromo-4-chloro-3-indolyl- $\beta$-D-galactopyranoside) indicator and methanolic or water-soluble fractions from alfalfa seed effusates when streptavidin $(6.2 \mathrm{mU})$ was present to bind any available biotin (data not shown).

To investigate bioS induction in experimental alfalfa rhizospheres, seeds were sterilized and inoculated with small numbers of Rm1021-WS44G cells carrying a bioS-gusA fusion. Staining 10- to 14-day-old plants with X-Gluc (5-bromo-

Table 1. The effect of biotin on transcription of a bioS-lacZ reporter fusion in Sinorhizobium meliloti RM1021-WSGB19

\begin{tabular}{lcc}
\hline & \multicolumn{2}{c}{$\beta$-galactosidase activity (Miller units) } \\
\cline { 2 - 3 } Growth phase & No biotin added & $\mathbf{4 0 ~} \mathbf{~ n M}$ biotin added \\
\hline Early log & $0.0 \pm 0.0$ & $3.1 \pm 1.2$ \\
Early stationary & $0.5 \pm 0.2$ & $12.6 \pm 0.3$ \\
Stationary & $2.1 \pm 0.3$ & $19.8 \pm 2.5$ \\
Late stationary & $\mathrm{ND}^{\mathrm{b}}$ & $26.1 \pm 0.1$ \\
\hline
\end{tabular}

${ }^{a}$ Data represent the mean \pm SD from three experiments.

${ }^{\mathrm{b}}$ Not determined. 
4-chloro-3-indolyl- $\beta$-D-glucuronic acid) indicator revealed that the bioS gene was strongly induced in bacteria growing on the alfalfa root (Fig. 4A). In addition, those bacteria that were growing on the agar near the root or the seed also showed bioS-gusA transcription (Fig. 4B), presumably suggesting that plant biotin was diffusing into the agar. No bioS-gusA transcription was observed in rhizobial colonies growing at a greater distance from the plant (Fig. 4B). This result complements a previous study that showed biotin is present in alfalfa root exudates (Rovira and Harris 1961). Finally, strong bioS-gusA expression also was observed in young alfalfa root nodules (Fig. 4C), thus suggesting a role for the BioS protein in symbiosis.

\section{Identification of the bioS promoter.}

In order to study the activity of the region identified by sequencing as the possible bioS promoter, three different bioSgusA fusions (Fig. 3) were mobilized into Rm1021, and bioS transcription was assayed at different biotin concentrations. The overall bioS-gusA transcription with the bioS-gusA-X fusion in extra copies in $S$. meliloti in pMB393 was significantly higher than the bioS transcription measured in strains Rm1021-WSG7 and Rm1021-WS44G, which carry only a single copy of the bioS-gusA fusion. The gusA activity observed for the bioS-gusA-H fusion was 11.3-fold lower at the $40 \mathrm{nM}$ biotin concentration tested, and the transcription of the
bioS-gusA-N was 15.1-fold lower than the activities observed for the bioS-gusA-X fusion (Table 2). A similar result was observed when $4 \mathrm{nM}$ biotin were present in the assay. However, the bioS transcription of the bioS-gusA-H was only 7.4-fold lower and the transcription measured for the bioS-gusA-N fusion was only 8.7-fold lower than bioS transcription observed for the bioS-gusA-X fusion. The increased biotin concentration present in the medium resulted in a 2.2 -fold increase in bioS transcription in the Rm1021bioS-gusA-X strain (Table 2). In contrast, the bioS transcription in the two strains carrying the bioS-gusA-N and the bioS-gusA-H fusion was only weakly affected by the increased amounts of biotin present in the test. In contrast to expression studies in which single copies of the bioS-gusA fusions were employed (Fig. 1), we observed already a relatively high bioS-gusA expression with the different bioS-gusA fusions supplied as extra copies when no biotin was added (Table 2). Taken as a whole, these results with the bioSgusA fusions in pMB393 indicate that the 129-bp region upstream from the ATG codon is important for promoter activity (Fig. 3; Table 2). However, the 444-bp DNA region upstream of the HindIII restriction site also is required for strong bioS promoter activity. When bioS-gusA activity was analyzed in the Escherichia coli background in the biotin auxotrophic strain EcWS-bioS-gusA-X in the presence of $40 \mathrm{nM}$ biotin, bioS transcription was only slightly above background. This
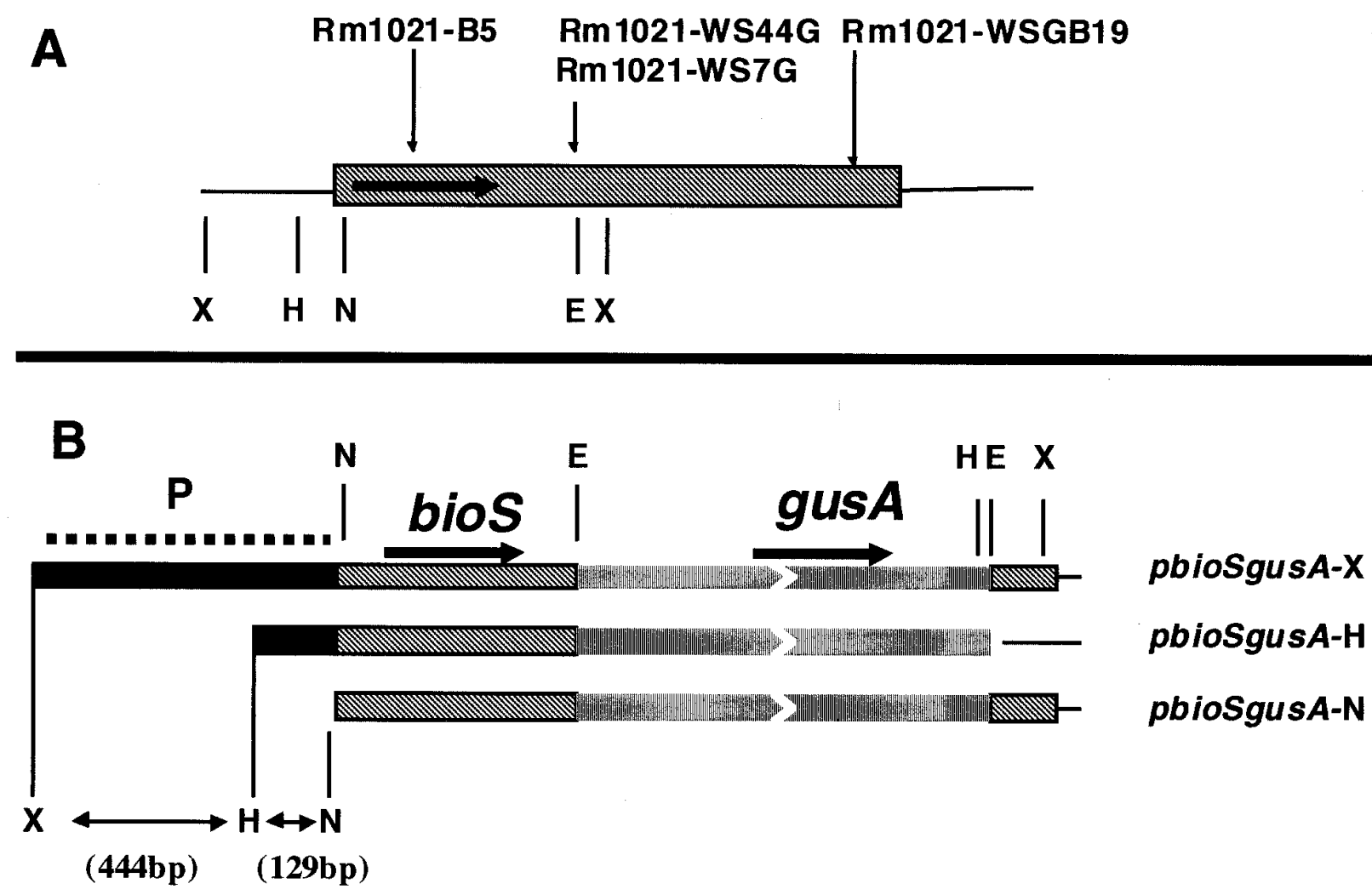

\section{$500 \mathrm{bp}$}

Fig. 3. Construction of bioS-gusA fusions in the medium copy vector pMB393 for a bioS promoter analysis. Horizontal arrow indicates direction of transcription in bioS. A, Vertical arrows indicate location of bioS transposon mutants and mutants created by homologous recombination used in this study. B, Construction of bioS-gusA promoter fusions pbioSgusA-X, pbioSgusA-H, and pbioSgusA-N in pMB393. P indicates bioS promoter as determined by expression studies and primer-extension experiments. DNA fragments were inserted into XhoI or HindIII restriction sites in pMB393. Correct orientation of insertions in pMB393 was confirmed by restriction analysis. Vertical lines mark restriction sites H, HindIII; N, NdeI; E, EcoRI; X, XhoI. 
low bioS transcription in the E. coli auxotrophic strain was independent from the biotin present in the medium (data not shown). However, this finding suggests that the bioS-gusA activity measured in $S$. meliloti with the same bioS-gusA fusion was indeed the result of the active promoter upstream of the bioS gene.

To further characterize the bioS promoter primer extension studies were performed. In these tests total RNA of Rm1021bioSgusA-X was isolated and hybridized with an excess of primer PE1 labeled with fluorescein prior to the reverse transcriptase reaction. In those experiments a transcriptional start site was identified as located 20/21 bp upstream of the ATG, as shown in Figure 5. A consensus ribosomal binding site was found 6 bp upstream of the ATG codon (Fig 5). Surprisingly, we identified a 7-bp TCCCCAT-motif homologue to the -10 region of the mucR -10 region within the bios -10 region and an 8-bp GCATTTGC-motif homologue to the $5^{\prime}$ region of the $b d h A-10$ region. This finding suggests that the three genes might underlie common regulatory principles.

\section{Overexpression and isolation of BioS protein.}

More than $95 \%$ of the $S$. meliloti BioS protein produced in E. coli BL21-EH13 was found as an insoluble pellet following centrifugation. When BioS was purified from the pellet under denaturing conditions, the protein was recovered in the manufacturer's buffer E, which is specified as normally eluting dimers or tetramers. Analysis of the purified BioS on $7.5 \%$ sodium dodecyl sulfate (SDS) polyacrylamide gels revealed an apparent molecular mass of $35 \mathrm{kDa}$ (Fig. 6A). Protein blot analyses with polyclonal antiserum produced in response to BioS protein indicated the BioS serum was quite specific for BioS protein with only minor cross-reactivity with other proteins in E. coli and S. meliloti (data not shown). As expected, BioS-specific bands detected in protein blots made with $E$. coli BL21-EH13 cell extracts and Rm1021 cell extracts were of equal sizes (Fig. 6B).

\section{BioS DNA and amino acid sequence analysis.}

Previous analyses established the absence of any significant homology in bios to other bacterial genes for either nucleotide sequence or amino acid sequence of proteins resulting from any of six possible reading frames (Streit and Phillips 1997). The molecular mass of $35 \mathrm{kDa}$ established here for BioS protein (Fig. 6) contrasts with the previously predicted mass of $22 \mathrm{kDa}$, which was estimated with the one predicted start codon that would allow expression of the inserted $n p t I I$ reporter gene (Streit and Phillips 1997). The validity of that particular start

Table 2. The effect of biotin on transcription of bioS-gusA reporter fusions in Sinorhizobium meliloti

\begin{tabular}{llcc}
\hline & \multicolumn{3}{c}{$\beta$-glucuronidase activity } \\
\cline { 2 - 4 } S. meliloti strain & $\begin{array}{c}\text { No biotin } \\
\text { added }\end{array}$ & $\begin{array}{c}\text { 4 nM biotin } \\
\text { added }\end{array}$ & $\begin{array}{c}\mathbf{4 0} \text { nM biotin } \\
\text { added }\end{array}$ \\
\hline Rm1021-bioS-gusA-X & $9,822(755)$ & $10,439(707)$ & $21,294(1,617)$ \\
Rm1021-bioS-gusA-H & $1,408(300)$ & $1,409(148)$ & $1,869(75)$ \\
Rm1021-bioS-gusA-N & $1,221(17)$ & $1,195(118)$ & $1,403(36)$ \\
\hline
\end{tabular}

a Data represent the mean \pm SD from three experiments.
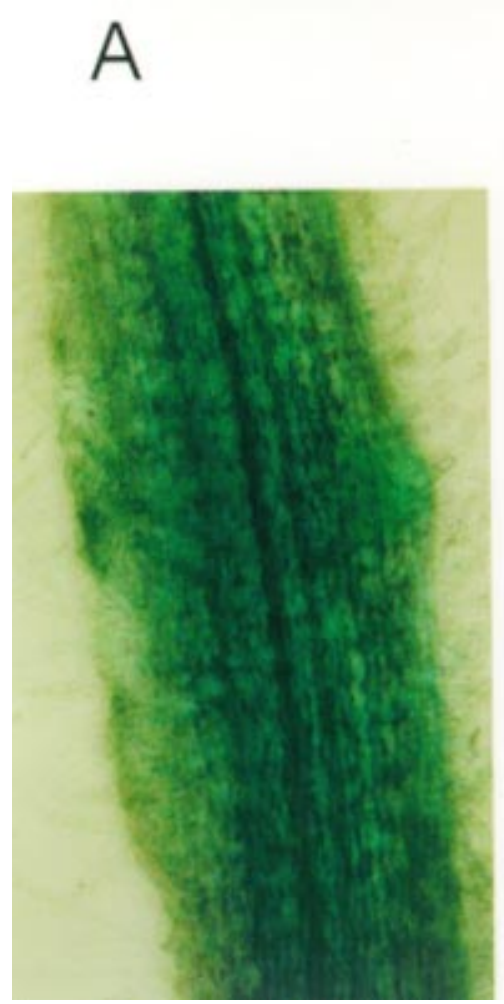
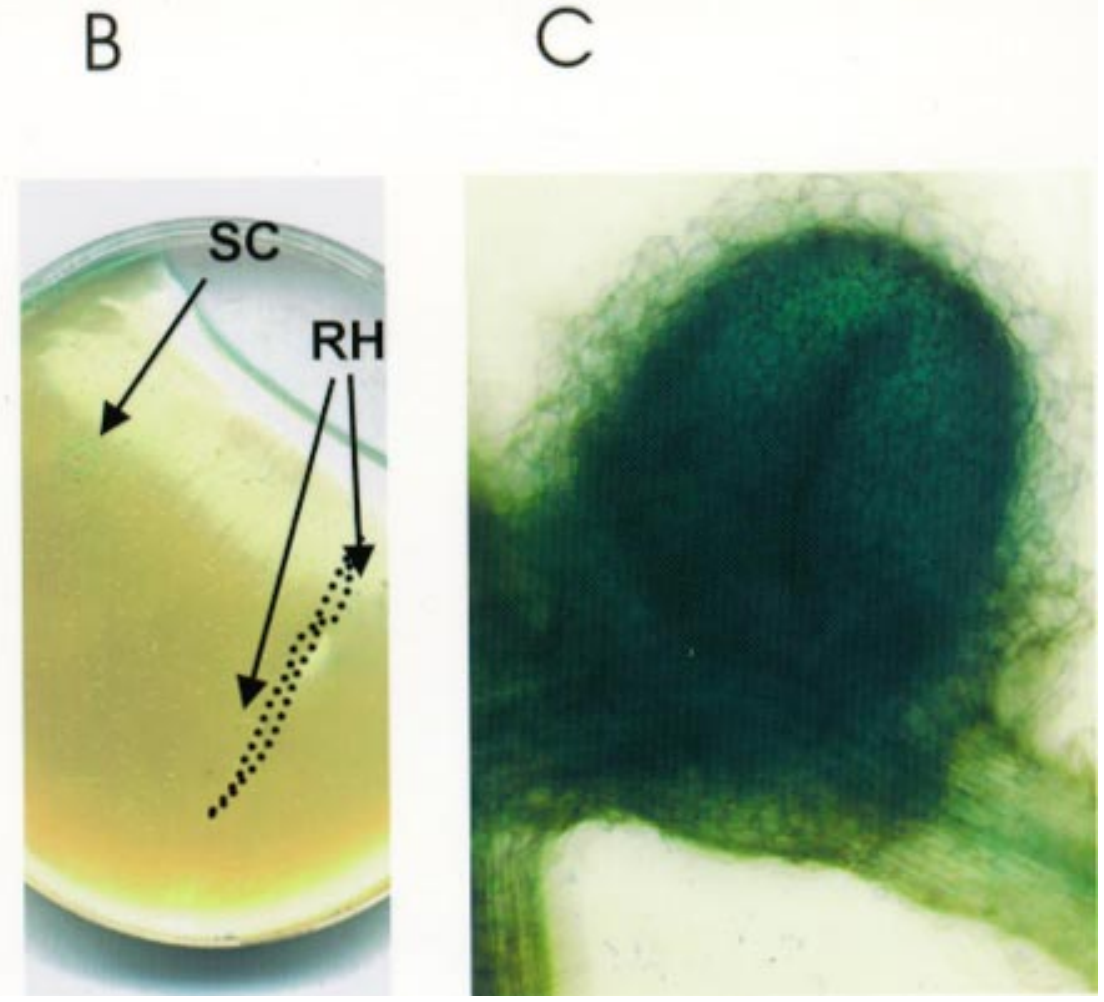

Fig. 4. Induction of bioS in Rm1021-WS44G cells by plant-exuded biotin. A, Magnification ( $\times 50)$ of alfalfa root after staining with X-Gluc (5-bromo-4chloro-3-indolyl- $\beta$-D-glucuronic acid). Control roots colonized and nodulated by Rm1021 were not stained by X-Gluc. B, Rhizobial colonies that had been growing in the rhizosphere $(\mathrm{RH})$ of a developing alfalfa root or close to an alfalfa seed coat (SC). Alfalfa seedling was removed from plate prior to staining with the $\beta$-glucuronidase substrate to prevent diffusion of blue color into the agar. Former position of seedling on plate indicated by a dotted line. C, Expression of a bioS-gusA reporter fusion in an alfalfa root nodule. 
codon is supported by analyses in the current study (Fig. 5). The $35-\mathrm{kDa} E$. coli protein in the current study was judged not to be a species artifact because the lac Z reporter in Rm1021WSGB19, which inserted downstream from the initially identified translational stop codon, shows the same biotin-dependent phenotype as any of the other bioS reporter mutants (Table 1,
Fig. 3). In addition, DNA analyses from two commercial laboratories did not identify a frame shift in the DNA sequence, which would result in a significantly larger open reading frame. Postulating an alternative codon usage for the first possible stop codon (TGA), which is located 597 nucleotides from the ATG codon, in the bioS open reading frame (GenBank no. U89162),

A

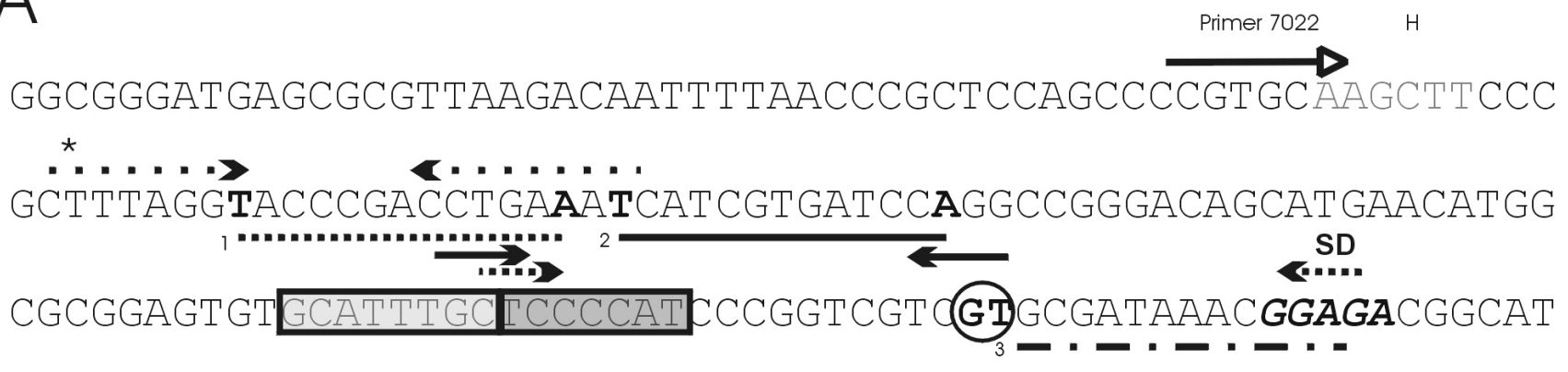

ATGCAGATCGAGAACA GGTT GAACGCT GCCGCT GCGT CAGGCGACGGCTTGGGCAAT
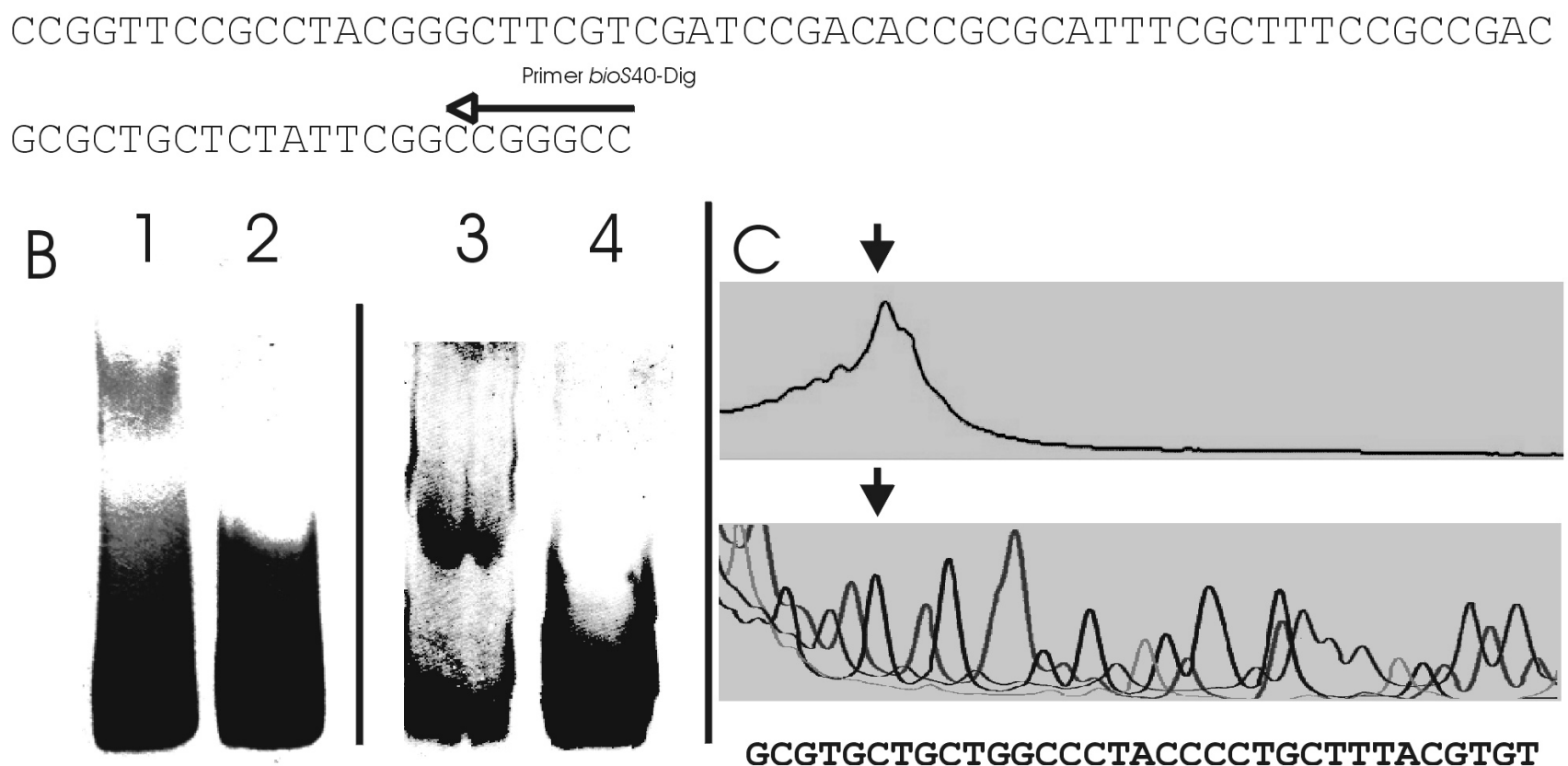

Fig. 5. The 5' DNA region of the bioS gene. A, DNA sequence containing the bioS promoter. Putative translational start codon is boxed; SD indicates position of consensus Shine-Dalgarno sequence (in bold italic letters). Transcriptional start site corresponds to a TG (circled, bold); bold T/A segments indicate the three T-N $\mathrm{N}_{(11)}$-A motifs. Motifs are also marked by a number (1-3) and underlined by a dotted (motif 1), solid (motif 2), or dashed (motif 3) line. Dyad structures corresponding to the different motifs are indicated by short horizontal arrows with dotted, solid, or dashed lines, respectively. $*=$ the dyad of the first T- $\mathrm{N}_{(11)}$-A motif is incomplete. B, BioS gel-retardation studies. Lane 1, BioS recombinant protein with DNA; lane 2, control reaction lacking DNA; lane 3, Rm1021 crude lysate with DNA; lane 4, control reaction lacking DNA; Lanes 1-2 and 3-4 represent two individual experiments analyzed on different gels; approximately 25 to $35 \mu \mathrm{g}$ of rhizobial protein extract and $5 \mu \mathrm{g}$ of BioS protein were used in gel-retardation studies. Primers 7022 and bioS40-Dig (A) were used to amplify the 321-bp, digoxigenin-labeled DNA fragment employed in the gel-shift assays. C, Mapping of the 5' end of bioS by a primer-extension reaction. Fluorogram (upper panel) shows primer-extension reaction, which produced a significant peak (vertical arrow) that corresponds to a T/G 20/21 bp upstream of the ATG codon as measured in a DNA sequencing reaction with the same primer (lower panel fluorogram). No such peak was detected in a control reaction (minus reverse transcriptase, data not shown). Reverse transcriptase reaction and sequencing reactions were performed with nonradioactively labeled primer PE1 (shaded horizontal arrow in A) and analyzed by automated sequencing. Primer extension experiment was conducted three times with the same result. TCCCCAT-motif homologue to the -10 region of the mucR (Bertram-Drogatz et al. 1997) -10 region is boxed and dark shaded; GCATTTGC-motif homologue to the $5^{\prime}$ region of the $b d h A$ (Aneja and Charles 1999 ) -10 region is boxed and light shaded (A). 
predicts a $34.5-\mathrm{kDa}$ protein consisting of 324 amino acids, a value similar to that observed in SDS polyacrylamide electrophoresis and immunological analyses (Fig. 6).

Several structural motifs in the DNA sequence upstream of the bioS gene and in the deduced amino acid sequence indicate that the BioS protein may belong to the LysR-type regulatory protein family: (i) The bioS promoter contains three nucleotide sequences consisting of a $\mathrm{T}-\mathrm{N}_{(11)}$ - A motif (Fig. 5A); (ii) Within these regions, dyadic nucleotide sequences possibly involved in protein binding and often associated with LysR-type receptor proteins are present (Fig 5A); and (iii) The deduced protein sequence contains homologies to the three different possible helix-turn-helix (HTH) DNA-binding motifs found in most LysR-type receptor proteins. Two of the motifs (I, II) were identified toward the $\mathrm{N}$ terminal region of the protein and only one in the $\mathrm{C}$ terminus (III). All three motifs were in the same reading frame. Both motifs detected in the N-terminal region of BioS were considered to be significant matches by the program (OWL25.3) used to identify the different motifs. Possible HTH motifs identified were NAAASGDGLGN (8) for motif I, TPRISLSADAL (49) for motif II, and LAFRQNGAAFNR (293) for motif III. Numbers in parentheses indicate the position of the first amino acid of the three different possible HTH motifs in the deduced BioS amino acid sequence.

\section{BioS-DNA binding studies.}

The hypothesis that BioS protein interacts with the LysRtype regulatory motifs in the promoter region of bioS was supported by results from BioS-DNA binding experiments. The electrophoretic mobility of a 321-bp, digoxigenin-labeled DNA fragment containing the $\mathrm{N}$-terminal region of the bioS gene and the promoter region (Fig. 5A) was definitely altered by the presence of BioS protein (Fig. 5). A low mobility BioSDNA complex was formed in the presence of extracts from cells that had been harvested in stationary phase from Rm1021 (Fig. 5B, lane 3). Recombinant BioS protein purified from E. coli BL21-EH13 also formed a low mobility complex with the bioS DNA (Fig. 5B, lane 1). These results support the hypothesis that the BioS protein in $S$. meliloti interacts with the 321-bp fragment used in the gel shift assay.

\section{Tests for bioS homologues in other bacteria.}

Heterologous DNA hybridization experiments showed that bioS is present in many $S$. meliloti strains but in few other bacteria tested (Table 3). Indeed, the bioS gene was detected only in the closely related species Sinorhizobium fredii. Tests with a DNA sequence specific for the putative $S$. meliloti survival operon surrounding bioS in $S$. meliloti detected strong hybridization in all $S$. meliloti strains and $S$. fredii. In addition, weak homologies were present in Rhizobium sp. strain NGR234 and Rhizobium sp. strain OR191. No DNA homologies were observed for either bioS or the putative survival operon in any of the other Gram-negative or Gram-positive soil microorganisms tested.

\section{DISCUSSION}

Results from these experiments establish that bioS is expressed most strongly in stationary-phase $S$. meliloti cells (Fig. 2, Table 1) and document that transcription of this gene is induced by biotin concentrations between 10 and $50 \mathrm{pM}$ (Fig. 1). These observations are consistent with the fact that open reading frames surrounding bioS in S. meliloti show high

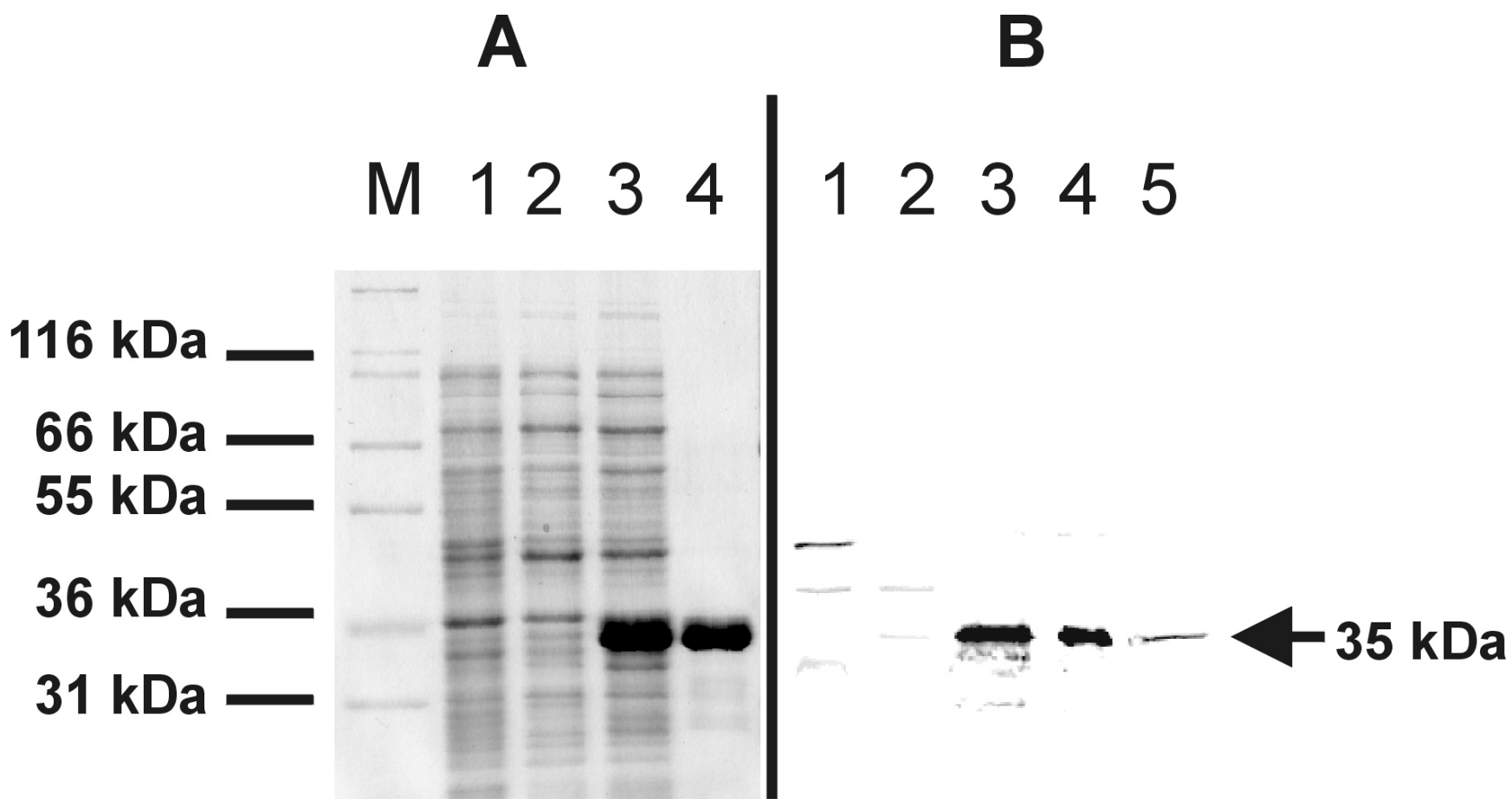

Fig. 6. Expression and immunological detection of a BioS histidine-tagged fusion protein. A, Sodium dodecyl sulfate (SDS) polyacrylamide gel electrophoresis analysis of recombinant BioS protein produced in Escherichia coli BL21-EH13 and crude cell extracts from Sinorhizobium meliloti. Cellular proteins were extracted and resolved on polyacrylamide gels. Lane M, protein size marker; lane 1, E. coli BL21; lane 2, E. coli BL21-EH13 prior to induction with isopropyl-beta-D-thiogalactopyranoside (IPTG); lane 3, E. coli BL21-EH13 $3 \mathrm{~h}$ after induction with $1 \mathrm{mM}$ IPTG; lane 4, $3 \mu \mathrm{g}$ of recombinant BioS protein after denaturing Ni-NTA purification. B, Immunological analysis of BioS. Lanes 1-4 as in A; lane 5, Crude extract of S. meliloti 1021 cells. 
homologies to genes found in prokaryotic survival operons and with the three phenotypes related to stationary phase that are observed in bioS mutants: (i) bioS mutants accumulate increased levels of biotin in stationary-phase cells; (ii) stationary-phase bioS mutants show an extended lag before resuming growth; and (iii) stationary-phase bios mutants compete poorly with wild-type cells in fresh medium under low-biotin conditions but grow equally well in the absence of biotin (Streit and Phillips 1997). The clear relevance of these phenotypes to successful growth in the rhizosphere and the demonstrated expression of bios on roots (Fig. 4A,B) reinforce the importance of biotin for $S$. meliloti colonization of alfalfa roots (Streit et al. 1996) and justify examination of the bioS promoter for a possible role in sensing plant-exuded biotin. The significance of bioS expression by bacteria in root nodules (Fig. 4C), which emphasizes a potential additional role for this gene in plant-microbe interactions, can be clarified only in future studies.

Several lines of evidence from this study support an initial conclusion that BioS is a regulatory protein involved in a transduction system that detects external biotin molecules. First, the finding that expression of a bioS-gusA fusion in the wild-type background (strain Rm1021-WS7G) was more than twice as high as that of the same reporter fusion in a bioS mutant background (strain Rm1021-WS44G) is consistent with the BioS protein functioning as a positive autoregulator of its own expression. Second, primer-extension experiments defined a promoter region that interacts with the BioS protein in gel-retardation studies (Fig. 5B) and thus are consistent with autoregulation of this locus. Third, analyses of the recombinant BioS protein showed many characteristics associated with an LysR-type transcriptional activator (Fig. 5A).

Expression studies with three bioS-gusA fusions (Fig. 3B) show clearly that the bioS promoter is located on a 573-bp fragment upstream of the ATG codon (Table 2). Primer-extension analysis confirmed that interpretation and identified a transcriptional start site 20/21 bp upstream of the ATG codon. Furthermore, DNA-gel retardation studies with BioS protein indicate that this protein interacts with a DNA region flanking the translational start codon of bioS (Fig. 5B). Thus, both structural and functional details of the bioS promoter are consistent with autoregulation of bioS by the BioS protein.

Structural evidence supporting a similarity between BioS and an LysR-type transcriptional activator comes from the DNA motifs identified in the bioS promoter region (Fig. 5A) and from the deduced amino acid sequence of BioS (Fig. 5C). In addition, most LysR-type receptors consist of approximately 300 amino acids, which is comparable to the size of the recombinant BioS protein expressed in $E$. coli and to the native protein in $S$. meliloti (Fig. 6). In the data bases used to locate possible helixturn-helix DNA-binding motifs in BioS, 111 LysR-type regulatory proteins are identified (Henikoff et al. 1988), including many nodD and syrM homologues in rhizobia (Kondorosi et al. 1991; Barnett et al. 1996). Most (93) of the 111 true LysR-type regulators contain three possible HTH motifs, and 18 contain two of the three binding motifs. The conserved HTH motifs consist of 11 or 12 amino acids completely encompassing the HTH motif (prosite pattern HTH-LysR-Family). All three possible DNAbinding motifs are present in BioS. Therefore, we speculate that the BioS protein may belong to the family of HTH-LysRtype receptors. The identification of three different $\mathrm{T}-\mathrm{N}_{(11)}-\mathrm{A}$ motifs and corresponding dyad structures in the bioS promoter region provides further evidence that BioS probably belongs to the LysR-type family of transcriptional regulators. Although it is believed that most LysR-type receptors interact with their co-repressor in the C-terminal domain, in no specific case has direct binding of the transcriptional regulator and the co-repressor molecule been shown (Schell 1993). Indeed, although it is reasonable to speculate that BioS binds biotin, we have no direct data supporting that possibility.

Several observations suggest that a more detailed examination of bioS may be informative. First, the bioS DNA locus apparently is the first biotin-regulated gene with no demonstrated link to biotin synthesis. This statement is based on the fact that cells grow well on defined medium without added biotin when they are mutated in the bioS locus studied here (Streit and Phillips 1997). Second, data base searches show no significant homologies for the bioS gene, and DNA hybridizations with a bioS-specific DNA probe suggest that the bioS gene is restricted to a very narrow range of bacteria (Table 3 ). Taken together, these observations suggest that bioS is an unusual gene that evolved in a small group of bacteria closely related to $S$. meliloti. Such uniqueness offers one possible explanation for the discrepancy between the previously postulated bioS open reading frame (Streit and Phillips 1997) and the actual size of the BioS protein measured in this work.

\section{MATERIALS AND METHODS}

\section{Bacterial strains and plasmids.}

Microbiological materials used in the present work are listed in Table 4. E. coli was grown at $37^{\circ} \mathrm{C}$ on Luria-Bertani

Table 3. DNA homologies of the bioS gene and the possible Sinorhizobium meliloti survival operon with total DNA isolated from 21 different bacteria

\begin{tabular}{lcc}
\hline & \multicolumn{2}{c}{ S. meliloti DNA probe } \\
specificity $^{\mathbf{b}}$
\end{tabular}


(LB) medium (Sambrook et al. 1989) supplemented with appropriate antibiotics. Rhizobia were cultured at $28^{\circ} \mathrm{C}$ on GTS (Kiss et al. 1979) or TY (Sambrook et al. 1989) medium, and growth was monitored as absorbance at $600 \mathrm{~nm}$.

\section{DNA manipulations, hybridization, and analyses.}

DNA was isolated and cloned by standard methods (Sambrook et al. 1989). DNA hybridizations were done with a nonradioactive digoxigenin DNA hybridization kit (Boehringer, Mannheim, Germany). The bioS-specific probe was derived as previously specified (Streit and Phillips 1997), and pbioS-SalI was used as a probe specific for the possible $S$. meliloti survival operon. In heterologous DNA analyses, hybridizations were done at $68^{\circ} \mathrm{C}$ for $S$. meliloti DNA and $62^{\circ} \mathrm{C}$ for all other species. DNA sequencing used protocols standard for ABI 377 automated DNA sequencers. Possible helix-turn-helix DNA-binding motifs were identified with the OWL 25.3 search software from the Biochemistry Department, University College London.

\section{Construction of bioS reporter strains.}

Transposon mutagenesis to make Rm1021-WSGB19 was performed by standard methods (Simon et al. 1989). The mutant was identified in PCR tests with Tn5-out and 7022 primers (Streit and Phillips 1997). Approximately 5,000 mutants were picked and analyzed. Putative mutants were confirmed by further PCR tests.
Plasmids for inserting gusA into bioS to make Rm1021WS7G and Rm1021-WS44 were constructed in the $s a c B$ positive selection suicide vector pJQ200mp18 (Quandt and Hynes 1993). A $3.3-\mathrm{kb}$ SalI fragment containing bioS was subcloned in pJQ200mp18 to form pWS7. A 2.0-kb fragment containing gusA from pCAM140 (Wilson et al. 1995) was excised with EcoRI and, after partial digestion of pWS7, inserted into the vector to produce pWS7G. The plasmid pWS7G was mobilized into Rm1021 in a triparental mating with pRK2013. Single recombinant clones were selected on GTS medium containing $40 \mathrm{nM}$ biotin, gentamicin $(50 \mathrm{mg} / \mathrm{liter})$, streptomycin (500 mg/liter), and X-Gluc (30 mg/liter). Double-recombinant clones were selected on the same medium in the presence of $5 \%$ sucrose. Insertion of the gusA gene in both mutant strains was analyzed by PCR with primers 7022 ( $5^{\prime}$ CCG TGC AAG CTT CCC GCT TTA GG $\left.3^{\prime}\right)$ and gusA-B (5' ACG TGC GTT CAT CCG GCG GGC CCT G 3').

Two of the three bioS-gusA promoter fusions in pMB393 (Fig. 3) were constructed by excising the HindIII or a XhoI fragment from pWS7G and inserting it into the corresponding sites in pmB393 to form pbioS-gusA-H or pbioS-gusA-X, respectively. For construction of pbioS-gusA-N the plasmid pbioS-gusA-X was restricted with NdeI and SmaI restriction enzyme, and the protruding $5^{\prime}$ end of the $N d e I$ restriction site was treated with $\mathrm{S} 1$ nuclease prior to religation. All constructs were mobilized into $R$. meliloti 1021 as described above and

Table 4. Microbiological materials

\begin{tabular}{|c|c|c|}
\hline Material & Relevant trait & Source or reference \\
\hline \multicolumn{3}{|l|}{ Escherichia coli } \\
\hline $\mathrm{DH} 5 \alpha$ & rec $\mathrm{A} 1, \Delta l a c Z$ & GibcoBRL, Karlsruhe, Germany \\
\hline VCS257 & Host strain for cosmid banks & Stratagene, La Jolla, CA \\
\hline HB101 & pRK2013 helper strain for matings & Stratagene \\
\hline K12 & Reference strain containing bio o peron & ATCC \\
\hline BL21(pLysS) & Host strain for protein expression & Novagen, Madison, WI \\
\hline BL21-EH1 & bioS gene in $\mathrm{pBSK}+$ in $\mathrm{DH} 5 \alpha$ & This work \\
\hline S17-1-pCAM140 & Promoterless gusA gene in mini $\operatorname{Tn} 5$ & Wilson et al. 1995 \\
\hline BL21-EH13 & bioS gene in pet $19 \mathrm{~b}$ (pet19b-bioS) in BL21(pLysS) & This work \\
\hline ATCC 33767 & bio operon deleted from $\lambda$ att-gal & ATCC \\
\hline ECWS-pMB393 & pMB393 in E. coli ATCC33767, control strain & This work \\
\hline ECWS-bioS-gusA-X & bioS-gusA-X in E. coli ATCC33767 & This work \\
\hline $\mathrm{S} 17-1$ & Modified RP4 plasmid integrated into genome & Simon et al. 1983 \\
\hline \multicolumn{3}{|l|}{ Sinorhizobium meliloti } \\
\hline Rm1021 & $2011, \mathrm{Sm}^{\mathrm{r}}$ & Meade et al. 1982 \\
\hline Rm1021-B5 & bioS::Tn5-B30 (nptII) mutant of Rm1021 & Streit and Phillips 1997 \\
\hline Rm1021-WSGB19 & bioS::Tn5-B22 (lacZ) mutant of Rm1021 & This work \\
\hline Rm1021-WS7G & bioS-gusA mutant of Rm1021 & This work \\
\hline Rm1021-WS44G & bioS-gusA mutant of Rm1021, double recombinant of Rm1021-WS7G & This work \\
\hline Rm1021-bioS-gusA-H & pbioS-gusA-H in Rm1021 & This work \\
\hline Rm1021-bioS-gusA-X & pbioS-gusA-X in Rm1021 & This work \\
\hline Rm1021-bioS-gusA-N & pbioS-gusA-N in Rm1021 & This work \\
\hline \multicolumn{3}{|l|}{ Plasmids } \\
\hline pBSK+ & pBluescript SK+ multicopy cloning vector & Stratagene \\
\hline pRK2013 & pRK212.2 derivative for matings & Figurski and Helinski 1979 \\
\hline $\operatorname{Tn} 5-\mathrm{B} 22$ & pSUP102 with transposable lacZ promoter probe, $\mathrm{Gm}^{\mathrm{r}}$ & Simon, et al. 1989 \\
\hline pet19B & Expression vector & Novagen \\
\hline pMB393 & Broad host range vector, $\mathrm{Spc}^{\mathrm{r}}$ & Gage et al. 1996 \\
\hline pbioS-gusA-H & bioS-gusA fusion in pMB393 (Fig. 4) & This work \\
\hline pbioS-gusA-X & bioS-gusA fusion in pMB393 (Fig. 4) & This work \\
\hline pbioS-gusA-N & bioS-gusA fusion in pMB393 (Fig. 4) & This work \\
\hline pbioS-SalI & bioS gene on a $3.3 \mathrm{~kb}$ SalI fragment in pBSK+ & Streit and Phillips, 1997 \\
\hline pJQ18mp200 & pACYC184ori, mob, $s a c B, \mathrm{Gm}^{\mathrm{r}}$ & Quandt and Hynes, 1993 \\
\hline pWS7 & pJQ18mp200 with bioS gene & This work \\
\hline pWS7G & pJQ18mp200 with bioS as gusA fusion & This work \\
\hline pEH1 & bioS gene in $\mathrm{pBSK}+$, in EcoRV site & This work \\
\hline pEH13 & bioS gene pet $19 \mathrm{~B}$, in $N d e \mathrm{I}$ site & This work \\
\hline
\end{tabular}


resulting strains were named Rm1021-bioS-gusA-N, Rm1021bioS-gusA-H and Rm1021-bioS-gusA-X.

\section{Cloning and expression of $\mathrm{BioS}$ as a histidine-tagged fusion protein.}

The bioS gene was amplified from $S$. meliloti chromosomal DNA with $P f u$ DNA polymerase in 35 cycles with primers bioS36a (5' GGG CAT ATG CAG ATC GAG AAC AGG TTG AAC GCT 3') and bioS36b (5' GGG GCA TAT GTT AAA GCG CAT TAG AGT TAC CGC $3^{\prime}$ ). Assuming that the bioS open reading frame (GenBank no. U89162) initially measured might not correlate with the size of the BioS protein, primers were designed to allow cloning of a much longer open reading frame consisting of $972 \mathrm{bp}$. The oligonucleotides were designed to introduce an NdeI site at both ends of the gene. The PCR fragments were ligated into $\mathrm{pBSK}^{+}$to make pEH1 and then excised with NdeI. This secondary fragment was ligated into pet $19 \mathrm{~b}$ to form $\mathrm{pEH} 13$. The expected nucleotide sequence of the PCR fragment in pet $19 \mathrm{~b}$ was confirmed by DNA sequencing. It revealed in this case that 2-bp exchanges had occurred in pEH13 at positions 15 and 16 downstream of the putative translational start codon that had no influence on the actual reading frame.

Freshly transformed E. coli BL21(pLysS) cells carrying pEH13 were used to overproduce the BioS protein. In cultures that had reached an optical density at $600 \mathrm{~nm}$ of 0.5 at $37^{\circ} \mathrm{C}$, production of the recombinant protein was induced by adding $1 \mathrm{mM}$ isopropylbeta-D-thiogalactopyranoside (IPTG). After $3 \mathrm{~h}$, cells were harvested, then disrupted in a French pressure cell, and BioS protein was purified from the soluble fraction and the pellet. Extracts were loaded on 1-ml Ni-nitrilotriacetic agarose (Ni-NTA) columns (Qiagen, Hilden, Germany), and chromatography was done as specified by Qiagen. Resulting protein fractions were analyzed by $7.5 \%$ SDS polyacrylamide gel electrophoresis.

\section{Primer extension studies.}

The bioS transcriptional start site was identified by automated fluorescent primer extension (Katzen et al. 1996) with a fluorescein-labeled primer (PE1) complementary to the $5^{\prime}$ terminus of the bioS gene. We used pbios-gusA-X as a control for the sequencing reaction and Rm1021-bios-gusA-X for RNA isolation. Total RNA was isolated with the RNAeasy kit (Qiagen) following the manufacturer's instructions.

\section{BioS antibody preparation and immunological detection.}

BioS-specific antibodies were obtained from Goetek (Göttingen, Germany). Purified BioS protein tagged with histidine at the N-terminal end was used for the injections. For Western blotting (immunoblotting) crude cell extracts and purified BioS protein were prepared, separated on 7.5\% SDS polyacrylamide gels and transferred onto nitrocellulose membranes (Sartorius, Göttingen, Germany). Primary rabbit antibodies were detected with alkaline-phosphatase-labeled secondary antibodies (Sigma, Heidelberg, Germany) with NBT (nitroblue tetrazoliumchloride) and BCIP (5-bromo-4-chloro3-indolyl phosphate) for colorimetric detection.

\section{BioS-DNA binding studies.}

Possible BioS-DNA interactions were examined in an electrophoretic mobility assay with a 321-bp DNA fragment containing the putative bioS promoter, which carried a nonradio- active label. Different dilutions of cell extracts or purified, histidine-tagged BioS protein were added to the digoxigeninlabeled DNA fragment $(0.5 \mu \mathrm{g} / \mu \mathrm{l})$ in a reaction buffer containing $0.4 \mathrm{mg}$ of bovine serum albumin (BSA) per $\mathrm{ml}, 50 \mathrm{mM}$ Tris $\mathrm{HCl}, \mathrm{pH} 8.0,100 \mathrm{mM} \mathrm{NaCl}, 0.05 \mathrm{mg}$ of sonicated herring testes DNA per $\mathrm{ml}$, and $5 \%$ glycerol. After incubation at $25^{\circ} \mathrm{C}$ for $30 \mathrm{~min}$, the reaction was loaded on a $5 \%$ polyacrylamide gel and electrophoresed 2 to $3 \mathrm{~h}$ at 40 to $50 \mathrm{~V}$ in $1 \times$ TAE buffer (Sambrook et al. 1989). The separated DNA and DNAprotein complexes were transferred to nylon membranes (Schleicher \& Schuell, Dassel, Germany) by vacuum blotting for $1 \mathrm{~h}$. Colorimetric detection of the digoxigenin-labeled DNA fragments was performed according to the protocol devised by Boehringer Mannheim. Primers used to amplify the 321-bp oligonucleotide for the nonradioactive band-shift tests were bioS40-Dig (5' CCG GCC GAG ATA GAG CAG CGC 3') and 7022. Positions of these primers are indicated in Figure 5. A PCR to amplify the 321-bp fragment from $S$. meliloti chromosomal DNA was run at $94^{\circ} \mathrm{C}$ for $1 \mathrm{~min}, 62^{\circ} \mathrm{C}$ for $1 \mathrm{~min}$, and $72^{\circ} \mathrm{C}$ for $1 \mathrm{~min}$. Reactions were done in standard PCR buffer in $50 \mu \mathrm{l}$. Following PCR amplification, the digoxigenin-labeled DNA fragment was electrophoresed on a $1.2 \%$ agarose gel and purified with the Qiaex II (Qiagen) gel extraction kit. Cell extracts of Rm1021 were prepared from stationary-phase cultures with a French pressure cell; recombinant BioS was purified as described below.

\section{$\beta$-Galactosidase and GUS assays.}

Activity of $\beta$-galactosidase and GUS was measured in cells harvested from cultures in GTS medium. When bioS transcription was measured at pM biotin concentrations, cells were transferred at least three times into fresh GTS medium without additional biotin prior to the experiment. All cultures were grown in 5-ml tubes, except for measurement of bioS transcription at different growth stages and bioS autoregulation, when 30-ml cultures in flasks were employed. All measurements were repeated at least three times by standard methods for $\beta$-galactosidase (Miller 1972) and GUS (Wilson et al. 1995) assays.

\section{Preparation of alfalfa seed effusates.}

Seed effusates were produced by soaking $50 \mathrm{~g}$ of alfalfa seeds (Medicago sativa cv. Europe) in $50 \mathrm{ml}$ of distilled water or methanol. After 2 days of soaking, the aqueous supernatant was filter sterilized and used in induction assays.

\section{Root colonization tests.}

bioS-gusA activity in alfalfa rhizospheres was assayed as described previously (Streit et al. 1992). Alfalfa plants (Medicago sativa cv. Moapa 69) for these tests were grown on agar plates containing one-half strength Hoagland medium (Hoagland and Arnon 1951) without nitrogen. Prior to inoculation, seeds were surface sterilized (Streit et al. 1996). In tests in which bioS-gusA activity of bacteria directly associated with the root surface was monitored, $50 \mu \mathrm{l}$ of bacterial cultures of Rm1021-WS44G was applied to each seed. When bacteria growing close to a developing root or germinating seed were assayed for bioS-gusA activity, $100 \mu \mathrm{l}$ of bacterial suspensions in fresh GTS medium containing approximately $1,000 \mathrm{CFU}$ per ml was spread on the plates. In those tests, plants were removed from the plates prior to being stained with X-Gluc to prevent diffusing of the blue dye into the agar. 


\section{ACKNOWLEDGMENTS}

This work was supported by a grant from the Deutsche Forschungsgemeinschaft (STR 451/2-1) and the Fonds der Chemischen Industrie. W. R. S. was in part supported by a stipend from the Deutsche Bundesstiftung Umwelt and the Alexander von Humboldt Stiftung. W. R. S. thanks A. Pühler and W. Liebl for helpful discussions.

\section{LITERATURE CITED}

Aneja, P., and Charles, T. C. 1999. Poly-3-hydroxybutyrate degradation in Rhizobium (Sinorhizobium) meliloti: Isolation and characterization of a gene encoding 3-hydroxybutyrate dehydrogenase. J. Bacteriol. 181:849-57.

Barker, D. F., and Campbell, A. M. 1981. Genetic and biochemical characterization of the birA gene and its product: Evidence for a direct role of biotin holoenzyme synthetase in repression of the biotin operon in Escherichia coli. J. Mol. Biol. 146:469-492.

Barnett, M. J., Rushing B. G., Fisher, R. F., and Long, S. R. 1996. Transcription start sites for syrM and nodD3 flank an insertion sequence relic in Rhizobium meliloti. J. Bacteriol. 178:1782-1787.

Bertram-Drogatz, P. A., Ruberg, S., Becker, A., and Pühler, A. 1997. The regulatory protein MucR binds to a short DNA region located upstream of the mucR coding region in Rhizobium meliloti. Mol. Gen. Genet. 254:529-38.

Bottomley, P. J. 1992. Ecology of Bradyrhizobium and Rhizobium. Pages 293-348 in: Biological Nitrogen Fixation. G. Stacey, R. H. Burris, and H. J. Evans, eds. Chapman and Hall, New York.

Cronan, J. E., Jr. 1989. The E. coli bio operon: Transcriptional repression by an essential protein modification enzyme. Cell 58:427-429.

Dunn, M. F. 1998. Tricarboxylic acid cycle and anaplerotic enzymes in rhizobia. FEMS Microbiol. Rev. 22:105-123.

Dunn, M. F., Encarnacion, S., Araiza, G., Vargas, M. C., Davalos, A., Peralta, H., Mora, Y., and Mora, J. 1996. Pyruvate carboxylase from Rhizobium etli: Mutant characterization, nucleotide sequence, and physiological role. J. Bacteriol. 178:5960-5970.

Figurski, D. H., and Helinski, D. R. 1979. Replication of an origincontaining derivative of plasmid RK2 dependent on a plasmid function provided in trans. Proc. Natl. Acad. Sci. USA 76:1648-1652.

Gage, D. J., Bobo, T., and Long, S. R. 1996. Use of green fluorescent protein to visualize the early events of symbiosis betweeen Rhizobium meliloti and alfalfa (Medicago sativa). J. Bacteriol. 178:7159-7166.

Henikoff, S., Haughn, G. W., Calvo, J. M., and Wallace, J. C. 1988. A large family of bacterial activator proteins. Proc. Natl. Acad. Sci. USA 85:6602-6606.

Hoagland, D. R., and Arnon, D. I. 1951. The Water Culture Method for Growing Plants without Soil. Vol. 347. University of California, Berkeley.

Katzen, F., Becker, A., Zorreguieta, A. Pühler, A., and Ielpi, L. 1996. Promoter analysis of the Xanthomonas campestris pv. campestris gum operon directing biosynthesis of the xanthan polysaccharide. J. Bacteriol. 178:4313-4318.

Kiss, G. B., Vincze, E., Kalman, Z., Forrai, T., and Kondorosi, A. 1979. Genetic and biochemical analysis of mutants affected in nitrate reduction in Rhizobium meliloti. J. Gen. Microbiol. 113:105-118.

Kondorosi, E., Pierre, M., Cren, M., Haumann, U., Buire, M., Hoffmann, B., Schell, J., and Kondorosi, A. 1991. Identification of NolR, a negative transacting factor controlling the nod regulon in Rhizobium meliloti. J. Mol. Biol. 222:885-896.

Li, S. J., and Cronan, J. E., Jr. 1993. Growth rate regulation of Escherichia coli acetyl coenzyme A carboxylase, which catalyzes the first committed step of lipid biosynthesis. J. Bacteriol. 175:332-340.

Miller, J. H. 1972. Experiments in Molecular Genetics. Cold Spring Harbor Laboratory, Cold Spring Harbor, NY.

Quandt, J., and Hynes, M. F. 1993. Versatile suicide vectors which allow direct selection for gene replacement in gram-negative bacteria. Gene 127:15-21

Rovira, A. D., and Harris, J. R. 1961. Plant root excretions in relation to the rhizosphere effect V. The exudation of B-group vitamins. Plant Soil 14:199-214.

Sambrook, J., Fritsch, E. F., and Maniatis, T. A. 1989. Molecular Cloning: A Laboratory Manual. 2nd ed. Cold Spring Harbor Laboratory, Cold Spring Harbor, NY.

Schell, M. A. 1993. Molecular biology of the LysR family of transcriptional regulators. Annu. Rev. Microbiol. 47:597-626.

Simon, R., Priefer, U., and Pühler, A. 1983. A broad host range mobilization system for in vivo genetic engineering: Transposon mutagenesis in gram-negative bacteria. Bio/Technology 1:784-791.

Simon, R., Quandt, J., and Klipp, W. 1989. New derivatives of transposon $\mathrm{Tn} 5$ suitable for mobilization of replicons, generation of operon fusions and induction of genes in gram-negative bacteria. Gene 80: 161-169.

Streit, W. R., Joseph, C. M., and Phillips, D. A. 1996. Biotin and other water-soluble vitamins are key growth factors for alfalfa root colonization by Rhizobium meliloti 1021. Mol. Plant-Microbe Interact. 9: 330-338.

Streit, W., Kosch, K., and Werner, D. 1992. Nodulation competitiveness of Rhizobium leguminosarum bv. phaseoli and Rhizobium tropici strains measured by use of the glucuronidase ( $g u s$ ) gene fusion. Biol. Fertil. Soils 14:140-144

Streit, W. R., and Phillips, D. A. 1997. A biotin-regulated locus, bioS, in a possible survival operon of Rhizobium meliloti. Mol. Plant-Microbe Interact. 10:933-937.

West, P. M., and Wilson, P. W. 1939. Growth factor requirements of the root nodule bacteria. J. Bacteriol. 37:161-185.

Wilson, K. J., Sessitsch, A., Corbo, J. C., Giller, K. E., Akkermans, A. D., and Jefferson, R. A. 1995. $\beta$-Glucuronidase (GUS) transposons for ecological and genetic studies of rhizobia and other gram-negative bacteria. Microbiology 141:1691-1705. 